\title{
BISp Informationen
}

Ger J Exerc Sport Res 2022 $52: 203-205$

https://doi.org/10.1007/s12662-022-00797-z

(c) The Author(s), under exclusive licence to Springer-Verlag GmbH Deutschland and Bundesinstitut für Sportwissenschaft, Deutscher Olympischer Sportbund, Deutsche Vereinigung für Sportwissenschaft 2022

Bundesinstitut für Sportwissenschaft
Bundesinstitut für Sportwissenschaft (BISp)

Lea Rossi

Fachbereich I - Forschung und Entwicklung

Graurheindorfer Str. 198

D-53117 Bonn

lea.rossi@bisp.de

www.bisp.de

\section{Einreichungen zur BISp- Antragsphase 2023}

Für die jährliche Antragsphase 2023 (Projektbeginn: 01.01.2023) wird die Frist zur Einreichung der Anträge auf den 31.05.2022 gesetzt.

Mit dieser Terminsetzung schafft das BISp einerseits einen angemessenen zeitlichen Abstand zur Bescheiderstellung der diesjährigen Antragsphase und berücksichtigt zum anderen die Auswirkungen der vorläufigen Haushaltsführung Anfang 2022.

\section{Neues Forschungsprojekt im Fachgebiet Sportanlagen}

Am 01. Januar 2022 startet im Bundesinstitut für Sportwissenschaft (BISp) ein neues Projekt zur Auswertung sportstättenbezogener Daten.

Anfang des Jahres 2021 wurde die Expertise Grundlagen für einen digitalen Sportstättenatlas unter der Projektleitung von Prof. Dr. Lutz Thieme von der Hochschule Koblenz veröffentlicht. Auf Grundlage dieser Expertise, einschließlich der durchgeführten Workshops, hat das Fachgebiet Sportanlagen im Juni 2021 ein Projekt mit dem Titel „Entwicklung und Validierung eines Verfahrens zur datenbasierten Ermittlung des individuellen Sanierungsbedarfs bundesdeutscher Sportstätten anhand des baulichen Zustands sowie zur Einschätzung des lokalen Versorgungsgrads mit Kernsportstätten" ausgeschrieben.
Den Zuschlag zur Durchführung erhielt ebenfalls die Hochschule Koblenz, Institut für Sportmanagement und Sportmedizinische Technik, unter der Projektleitung von Prof. Dr. Lutz Thieme. Die Beauftragung sieht vor, dass ein Verfahren zur einheitlichen Auswertung verschiedener Datenbestände unterschiedlicher Herkunft, Struktur und Ausprägung entwickelt wird.

Einige Bundesländer und einzelne Kommunen verfügen bereits über umfangreiche Datenbanken zu Sportstätten, die auf die lokalen, spezifischen Bedarfe und Besonderheiten der dort vorhandenen Sportinfrastruktur eingehen. Diese werden als Grundlage zur besseren Steuerung und Kontrolle von Investitionen beispielsweise zur lokalen Sport- und Sportstättenentwicklungsplanung, zur Priorisierung von Sanierungs- und Neubauvorhaben, zur Anpassung an sich verändernde Bedarfe oder auch bei kurzfristig auftretenden Problemstellungen, genutzt. Daten zu Sportstätten können zur Optimierung der Auslastung einer Sportstätte durch die sportlich Aktiven oder zur nachhaltigen, möglichst umweltschonenden Verwendung von Baustoffen beim Bau und bei der Sanierung oder zum effizienten Einsatz von Energieträgern im Betrieb von Sportstätten genutzt werden.

Bei der Bearbeitung ist die Hochschule Koblenz auf die Bereitschaft zur Mitwirkung einzelner Bundesländer und Kommunen angewiesen, damit sie anhand von vorhandenen $\mathrm{Da}$ tensätzen ein valides Verfahren entwickeln und zur Verfügung stellen kann. Eine auf möglichst alle derzeitigen und zukünftigen Datensammlungen anwendbare, einheitliche Methode zur Auswertung würde vor dem Hintergrund der jeweiligen Versorgungsgrade schnell $\mathrm{zu}$ einer objektiven, validen und anerkannten Grundlage zur Ermittlung und Bewertung von Förderbedarfen im Sportstättenbau in der Planung, im Bau, im Betrieb, zum baulichen Zustand und zur Weiterentwicklung führen. Bund, Bundesländer und Kommunen ständen somit Entscheidungshilfen für Erhalt, Sanierung und Ausbau einer bedarfsgerechten, flächendeckenden Sportinfrastruktur zur Verfügung.

Insbesondere die Entwicklung eines Verfahrens zur Erfassung des baulichen Zustands von Sportstätten wird von verschiedenen Parametern beeinflusst und kann je nach Lokalisation der Sportstätte sehr unterschiedlich sein. Das Forschungsprojekt soll hier Wege aufzeigen, wie ggf. ein Verfahren zur datenbasierten Auswertung von Informationen über Sportstätten auch in Bezug auf den Digitalen Sportstättenatlas Deutschland (DSD) eine valide Einschätzung des baulichen Zustands von Sportstätten ermöglichen kann, bzw. welche Daten in welcher Anzahl und Qualität hierfür benötigt werden.

Die Laufzeit des Projekts ist für zwei Jahre, bis zum 31.12.2023, angesetzt.

Die Veröffentlichung zur Expertise „Grundlagen für einen digitalen Sportstättenatlas“ finden Sie unter folgendem Pfad: www.bisp.de $\rightarrow$ Wissenstransfer $\rightarrow$ BISp-Publikationen 


\section{Aufbau einer Datenplattform des Bundes für deutsche Sportstätten}

Zur Deckung von bundeseigenen Informationsbedarfen aus Politik und Verwaltung entwickelt das Bundesinstitut für Sportwissenschaft (BISp) eine Datenplattform „Digitaler Sportstättenatlas für Deutschland“ (DSD). Damit sollen alle Kernsportstätten (Bäder, Sporthallen und Sportplätze) in der Bundesrepublik Deutschland lokalisiert und identifiziert werden.

Mögliche Einsatzgebiete der Plattform liegen beispielsweise in der Beantwortung von Kleinen Anfragen aus dem parlamentarischen Raum als auch in der Bereitstellung von unterstützenden Informationen und Forschungsdaten für Forschungsnehmende im Rahmen der Förderung von wissenschaftlichen Projekten durch das BISp. Eine Erfassung der spezifischen Sanierungsbedarfe beziehungsweise der baulichen Zustände einzelner Sportstätten ist nicht vorgesehen.

Die Entwicklung der ersten Ausbaustufe der Plattform soll planmäßig zum 31.12.2022 abgeschlossen werden. Nach Bedarfslage des Bundes ist für die nachfolgenden Jahre die Weiterentwicklung hinsichtlich zusätzlicher Ausbaustufen sowie der funktionale Ausbau der Plattform geplant.

Zur Beantwortung sportpolitischer Fragestellungen aus Datensammlungen zu Sportstätten startet das BISp am 1. Januar 2022 ein gesondertes Forschungsprojekt.

\section{Neuer BISp-Podcast „Athlete Performance Profiling im Anti- Doping"}

Im Rahmen des Projekts „Wissenschaftlicher Umgang mit kleinen Stichproben bis hin zu Single-Subject-Designs" sollten die zusammengetragenen Erkennt-

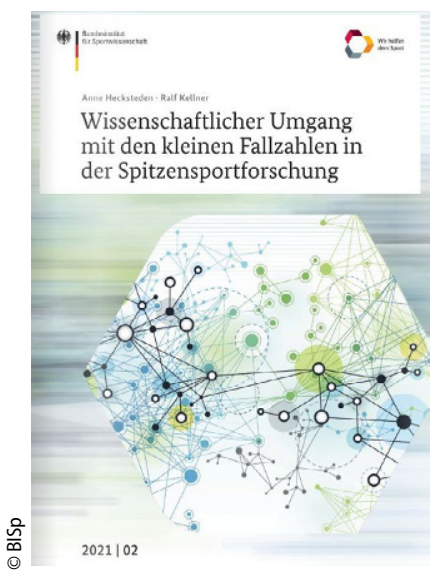

Wonneberger (Fachgebietsleiter Dopingbekämpfung, BISp) mit Frau PD Dr. Anne Hecksteden (Projektleiterin, Universität des Saarlandes) und Stefan Trinks (Ressortleitung Dopingkontrollsystem, NADA).

Den Podcast ist auf dem YouTube-Kanal des BISp verfüg- bar (https://www.youtube.com/ watch?v=4QtxPRMIyNw).

Die Veröffentlichung „Wissenschaftlicher Umgang mit den kleinen Fallzahlen in der Spitzensportforschung" finden Sie unter folgendem Pfad: www.bisp.de $\rightarrow$ Wissenstransfer $\rightarrow$ BISp-Publikationen

\section{Studie zu ökonomischen Effekten der COVID-19- Pandemie auf Athletinnen und Athleten liegt vor}

Ein Forschungsteam unter der Leitung von Prof. Dr. Christoph Breuer (Deutsche Sporthochschule Köln) hat die Effekte der Pandemie auf die Einkommenssituation von Sportlerinnen und Sportlern erhoben, die von der Stiftung Deutsche Sporthilfe finanziell gefördert werden.

Daraus konnten u. a. Erkenntnisse abgeleitet werden, inwieweit Sponsoren- und sonstige Einnahmen während der vergangenen Monate zurückgegangen sind.

Die Veröffentlichung „Die ökonomischen Auswirkungen von COVID-19 für Nachwuchsleistungs- und Spitzensportlerinnen und Spitzensportler in
Deutschland" finden Sie unter folgendem Pfad: www.bisp.de $\rightarrow$ Wissenstransfer $\rightarrow$ BISp-Publikationen

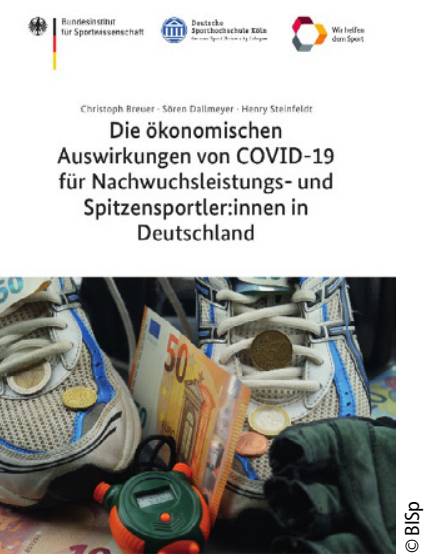

nisse zum Umgang mit kleinen Fallzahlen in praxisnahen Anwendungsfelder im Sinne eines "proof-of-concept" überprüft werden. Der neue BISp-Podcast zeigt ein beispielhaftes Anwendungsfeld auf.

Eines dieser Anwendungsfelder wurde in Zusammenarbeit mit der Nationalen Anti Doping Agentur Deutschland (NADA) bearbeitet. Hierbei wurde ein erster Algorithmus-Dummy auf Basis öffentlich zugänglicher Daten entwickelt, welcher die Entdeckung auffälliger leistungsbezogener Entwicklungsverläufe ermöglichen soll.

Über diesen noch recht jungen Ansatz im Bereich des AntiDopings unterhält sich Dr. Marc

\section{"Sportwirtschaft - Fakten \& Zahlen“: Ausgabe 2021 verfügbar}

Auf Basis der Befunde des vom Bundesministerium für Wirtschaft und Energie (BMWi) und Bundesinstitut für Sportwissenschaft (BISp) geförderten Forschungsvorhabens „Monitoring Sportsatellitenkonto" hat das
BMWi eine neue Broschüre veröffentlicht.

Die Veröffentlichung „Sportwirtschaft: Fakten \& Zahlen Ausgabe 2021" finden Sie unter folgendem Pfad: www.bisp.de $\rightarrow$ Wissenstransfer $\rightarrow$ BISp-Publikationen 


\section{WVL-Ausschreibungsprojekt "Leistungsreserve Individu- alisierung": Forschungskon- sortium um Prof. Dr. Karsten Krüger erhält Zuschlag zur Förderung}

Auf der Basis einer umfangreichen Begutachtung und einhergehenden Beratung zu den eingegangenen Forschungsanträgen hat sich der Projektantrag des Konsortiums um Herrn Prof. Krüger (Universität Gießen) gegen die der Mitbewerberinnen und Mitbewerber durchsetzen können. Das Projekt wird nun zeitnah an die Projektgruppe vergeben.

Mitantragstellende sind Prof. Dr. Karen Zentgraf (Universität Frankfurt), Prof. Dr. Lena Wiese (Universität Frankfurt), Prof. Dr. Michael Mutz (Universität Gießen) und Prof. Dr. Dr. Markus Raab (DSHS Köln).
Zum derzeitigen Zeitpunkt sind zudem zahlreiche Praxispartner mittels einer Kooperationsvereinbarung in das Projekt eingebunden:

Spitzenverbände: Deutscher Volleyball-Verband, Deutscher Turner-Bund, Deutscher Eishockey-Bund, Deutscher BasketballBund, Bob und Schlittenverband für Deutschland, Deutscher Verband für Modernen Fünfkampf

Olympiastützpunkte: Bayern, Berlin, Brandenburg, RheinNeckar, Hessen, Niedersachsen, Stuttgart

Der Projektstart wird voraussichtlich der 01.12.2021, ggf. der 01.01.2022 sein.

\section{Weiterentwicklung des „Wissensmanagements im WVL" - Kick-Off-Veranstaltung für die Umsetzung erfolgreich gestartet}

In einer digitalen Kick-Off-Veranstaltung erfolgte am 25. Oktober 2021 der Startschuss für die Ausgestaltung und Umsetzung der bislang im Wissenschaftlichen Verbundsystem Leistungssport (WVL) erarbeiteten Ziele, Aufgaben und Visionen zur Weiterentwicklung des Wissensmanagements im WVL.

Die Einladung des Deutschen Olympischen Sportbundes (DOSB) und des Bundesinstituts für Sportwissenschaft (BISp) nah- men über 60 Teilnehmende aus dem WVL-Netzwerk an, um über die schrittweise Implementierung der in den WVL-Workshops erzielten Ergebnisse sowie über weitere Meilensteine zu beraten. Das BISp und der DOSB freuen sich auf die weitere Zusammenarbeit mit den WVL-Partnern im Wissensmanagement-Netzwerk, um gemeinsam dem deutschen Leistungssport dauerhaft das beste Wissensmanagementsystem zur Verfügung stellen zu können. 